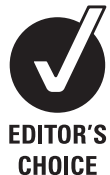

CHOICE
${ }^{1}$ Department of Emergency Medicine, Cathay General Hospital, Taipei, Taiwan ${ }^{2}$ School of Medicine, Fu-Jen Catholic University, Taipei, Taiwan

${ }^{3}$ Institute of Aerospace Medicine, School of Medicine, National Defense Medical Center, Taipei, Taiwan ${ }^{4}$ Department of Neurology, Tri-Service General Hospital, Taipei, Taiwan

\section{Correspondence to}

Dr Hsin Chu, Institute of Aerospace Medicine, School of Medicine, National Defense Medical Center, No. 161 Section 6, Min-Chuan East Road, Taipei, 114 Taiwan, R.O.C.:

hrchu@mail.ndmctsgh.edu.tw

Accepted 29 October 2010 Published Online First 25 February 2011

\title{
Initial salivary $\alpha$-amylase activity predicts malignant ventricular arrhythmias and short-term prognosis after ST-segment elevation myocardial infarction
}

\author{
Ying-Sheng Shen, ${ }^{1,2}$ Chia-Meng Chan, ${ }^{1,2}$ Wei-Lung Chen, ${ }^{1,2}$ Jiann-Hwa Chen, ${ }^{1,2}$ \\ Hsin-Yu Chang, ${ }^{1} \mathrm{H} \sin \mathrm{Chu}^{3,4}$
}

\begin{abstract}
Background Ventricular arrhythmias (VA), including ventricular tachycardia and ventricular fibrillation, are the most common remediable cause of death in patients with acute myocardial infarction. Augmented

sympathetic neural activity to the heart and myocardial catecholamine release may be the primary factors in the generation of VA. The aim of this study was to assess the predictive value of salivary $\alpha$-amylase (sAA) activity, an indicator of sympathetic activity, for malignant VA occurrence and for short-term outcome in patients with ST-segment elevation myocardial infarction (STEMI).

Methods Patients with STEMI $(n=91)$ were recruited from the Emergency Department during the period 1 December 2008 to 31 April 2010. Correlations of initial sAA activity at presentation with VA, as well as 1-month prognosis were analysed.
\end{abstract}

Results SAA activity was significantly increased in the VA group (395 $\pm 173.7 ; n=9$ ) as compared with the nonVA group (283 $\pm 89.3 ; n=82)(p=0.014)$. The adjusted OR for malignant ventricular arrhythmia occurrence was 1.010 (95\% Cl 1.001 to 1.018). Eight patients (8.8\%) died and 24 (26.4\%) had at least one short-term adverse event within the first month after STEMI. Simple logistic regression analysis showed that $\mathrm{SAA}$ is an independent predictor for short-term prognosis ( $p=0.049$, OR 1.005, 95\% Cl 1.000 to 1.009).

Conclusions Although a prospective study with a large cohort is required, the present results suggest that high initial SAA activity is associated with increased risk of malignant VA and predicts short-term prognosis in patients with STEMI.

\section{INTRODUCTION}

Sudden cardiac death (SCD) is a major cause of morbidity and mortality in patients with coronary artery disease and myocardial infarction (MI). Despite significant breakthroughs in the treatment of acute myocardial infarction (AMI), early mortality is still high. Ventricular arrhythmias (VA), including ventricular tachycardia and ventricular fibrillation, are the most common remediable cause of death in patients with AMI, and it is clearly important that they should be predicted and thereby prevented. ${ }^{12}$ The incidence of VA has been reported to be $1.9 \%$ to $10.2 \%$ in all AMI patients, and to be highest in the early hours after AMI. ${ }^{1} \quad 3 \quad 4$ Although the pathogenesis and precursors of VA may well differ depending on the time after onset of infarction that the arrhythmia occurs and the clinical context, it is well known that the activity of the autonomic nervous system is an important factor in the initiation of VA during AMI. ${ }^{5}$ Previous studies have reported that augmented sympathetic neural activity to the heart and myocardial catecholamine release may be the primary factors in the generation of VA. ${ }^{6}$ Activation of the sympathetic nervous system (SNS) contributes to the occurrence of ischaemia-induced VA, whereas the parasympathetic nervous system has an antiarrhythmic effect in the ventricles. ${ }^{7-9}$ Moreover, epidemiological studies suggest that in approximately $25 \%$ of patients, psychological stress precedes sudden death due to coronary artery disease. ${ }^{10} 11$ Acute emotional stress, a known activator of the SNS, is associated with left ventricular contractile dysfunction, myocardial ischaemia, disturbances of cardiac rhythm, hospital-acquired infection and mortality in patients after MI.

Recently, there has been increased interest in the use of oral fluid samples for diagnosis of a variety of diseases. Neuroendocrine markers, such as salivary $\boldsymbol{\alpha}$-amylase (sAA), play an important role as indicators of the magnitude of human responses to acute stressful events from various physiological and psychological stimuli, and as markers of SNS. ${ }^{12}$ The salivary gland, glandular duct cells and vascular bed of the salivary glands are rich in $\beta$ adrenoreceptors. The action of norepinephrine released from sympathetic nerve terminals on adrenergic receptors increases the activity of the salivary glands, thus causing an increased protein-to-fluid ratio in the saliva. Among these proteins is SAA, an enzyme that is produced by the salivary gland. sAA activity has been suggested to reflect catecholaminergic changes $^{13}$ and therefore might serve as a noninvasive and easily obtained surrogate marker of SNS activation.

The aim of this study was to examine whether sAA activity level has predictive power for malignant ventricular arrhythmia in patients with STsegment elevation myocardial infarction (STEMI) in the Emergency Department (ED). It was also aimed to assess whether sAA activity level could be used as a prognostic factor for short-term prognosis in these patients.

\section{METHODS}

\section{Study design}

This was a prospective, observational study designed to assess whether initial sAA activity could predict malignant VA in patients with acute STEMI in the ED. The study protocol has been 
approved by the Institutional Review Board for Human Research of the Cathay General Hospital, and written or oral informed consents were obtained from the patients.

\section{Study setting and population}

Data were collected on all consecutive patients from December 2008 to April 2010 with a diagnosis of STEMI according to the current European Society of Cardiology/American College of Cardiology definition. ${ }^{14}$ Patients were excluded according to the following criteria: oral illness and acute coronary syndromes diagnosed at another hospital and referred for further treatment. Malignant ventricular arrhythmia was defined as ventricular fibrillation or ventricular tachycardia with haemodynamic compromise requiring defibrillation. Patients were categorised into one of two study groups, VA and non-VA attack during the ED stay. Diabetes mellitus was defined as when the patient reported a history of diabetes or was receiving treatment with an oral hypoglycaemic agent or insulin. Current or former smokers were recorded as 'smoker'. The composite short-term adverse events included the occurrence of at least one of the following events during the hospital stay: 1-month mortality; cardiogenic shock, defined by a systolic blood pressure of less than $90 \mathrm{~mm} \mathrm{Hg}$ and a prolonged hypotensive episode with poor peripheral perfusion that was not responsive to fluids or that required institution of inotropic infusions to maintain blood pressure; VA; congestive heart failure, defined by dyspnoea with concurrent interstitial oedema on the chest radiograph; and hospital-acquired infection. To assess the predictors for shortterm prognosis, the patients were classified into two groups according to the presence or absence of short-term adverse outcome. Demographic characteristics of the patients, their vital signs at triage and their clinical history, as well as results of initial laboratory and image studies, were collected by the attending ED physician and recorded on standardised patient data collection sheets. The outcomes of the patient were blinded to the investigators performing the sAA analysis.

\section{Collection and measurements of samples}

Saliva samples were obtained by unstimulated passive drool into a Salivette saliva collecting tube (Sarstedt, Nümbrecht, Germany) at inclusion. Before each sample collection, patients were told to rinse their mouth with water. Samples were stored immediately after collection at $0^{\circ} \mathrm{C}$ and delivered to the laboratory the next day, where samples were frozen at $-80^{\circ} \mathrm{C}$ until analysis. The sAA activity was measured with a sAA quantitative enzyme kinetic colorimetric assay kit (Salimetrics, Pennsylvania, USA).

\section{Statistical analysis}

Data are reported according to the specifications provided in the 'Standardized Reporting Guidelines for Studies Evaluating Risk Stratification of ED Patients with Potential Acute Coronary Syndromes', 15 and are presented as mean \pm SD or frequency (percentage) when appropriate. Categorical variables were compared between two groups using the $\chi^{2}$ test or Fisher exact test as appropriate, whereas continuous variables were compared using the Mann-Whitney $U$ test. The variables with a univariate comparison $\mathrm{p}<0.05$ between two groups were eligible for inclusion in an entry selection multiple logistic regression model that identified the clinical factors in the ED that were independent predictors of malignant VA in this patient cohort. To determine the accuracy of sAA level for predicting malignant VA and short-term adverse events, the receiver operating characteristic (ROC) curves and their corre- sponding areas under the curve were used. A $p$ value less than 0.05 was considered statistically significant. A common statistical package (SPSS 17.0 for Windows, SPSS Inc.) was used to perform all statistical tests.

\section{RESULTS}

During the 16-month period of the study, 93 patients were admitted to the ED with STEMI, and 91 patients (71 men and 20 women; mean age $64 \pm 11$ years) were eligible for inclusion in the study. Of the 93 patients, two patients were excluded because they did not produce enough saliva for analysis. Of the study patients, $61.5 \%$ suffered anterolateral infarction and $38.5 \%$ inferolateral infarction. The incidence of malignant VA in the 91 patients was $9.9 \%(n=9)$, seven $(7.7 \%)$ of which were cases of ventricular fibrillation and two $(2.2 \%)$ of sustained ventricular tachycardia. The mean duration from ED arrival to the development of VA was $27 \pm 16.1 \mathrm{~min}$. Of the 91 patients included in the study, eight (8.8\%) died and 24 (26.4\%) had at least one short-term adverse event within first month after STEMI. Patient characteristics stratified by the occurrence of VA after STEMI are summarised in table 1. There were no significant differences in sex, initial heart rate, smoking, diabetes mellitus, infarct sites, initial white cell count and glucose level on admission between these two groups of patients. Prior history of MI, and initial CK-MB and troponin I levels were not different between the two groups ( $p=0.581,0.873$ and 0.694 respectively; data not shown). In contrast, younger age, a lower serum potassium level and a higher sAA activity level were associated with an increased risk of malignant VA.

Multiple logistic regression models were performed to analyse the risk of post-STEMI VA (dependent variable) associated with the following independent variables: age, serum potassium concentration and sAA activity. The results showed that higher sAA activity levels and younger age were independent significant predictors of the occurrence of malignant VA in patients (table 2). In the ROC curve of sAA activity in predicting postSTEMI malignant VA (figure 1), the area under the curve was found to be 0.749 (95\% CI 0.519 to 0.980 ).

To evaluate the predictor of short-term prognosis after STEMI, the patients were stratified into two groups according to the presence or absence of short-term adverse events (table 3 ). Simple logistic regression analysis showed that sAA is an independent predictor for short-term prognosis $(p=0.049$, OR 1.005

Table 1 Demographic and clinical characteristics of the ventricular arrhythmia (VA) and non-VA groups

\begin{tabular}{llll}
\hline & $\begin{array}{l}\text { VA group } \\
(\mathbf{n = 9})\end{array}$ & $\begin{array}{l}\text { Non-VA group } \\
(\mathbf{n = 8 2})\end{array}$ & p value \\
\hline Age (years) & $55 \pm 6.6$ & $65 \pm 11.5$ & $0.009^{*}$ \\
Gender, male/female, $\mathrm{n}(\%)$ & $8 / 1$ & $63 / 19$ & 0.367 \\
Smoker & $6(66.7)$ & $44(53.7)$ & 0.210 \\
Diabetes mellitus & $1(11.1)$ & $12(14.6)$ & 0.622 \\
Initial heart rate (bpm) & $80 \pm 17$ & $79 \pm 18$ & 0.755 \\
Laboratory data & & & \\
$\quad$ WBC (per cm ${ }^{3}$ ) & $11.4 \pm 4.05$ & $10.5 \pm 3.00$ & 0.325 \\
$\quad$ Glucose (mg/dl) & $162 \pm 80.2$ & $153 \pm 66.9$ & 0.989 \\
$\quad$ Serum potassium (mEq/l) & $3.6 \pm 0.33$ & $3.9 \pm 0.35$ & $0.034^{*}$ \\
$\quad$ sAA activity (U/ml) & $395 \pm 173.7$ & $283 \pm 89.3$ & $0.014^{*}$ \\
Anterior infarction, $\mathrm{n}(\%)$ & $6(66.7)$ & $49(59.8)$ & 0.367 \\
\hline
\end{tabular}

Continuous variables are presented as mean \pm SD.

Percent values are in parenthesis.

${ }^{*} \mathrm{p}<0.05$ between two groups.

sAA, salivary $\alpha$-amylase; WBC, white blood cell. 
Table 2 Multiple logistic regression analysis showed independent predictors for malignant VA

\begin{tabular}{lll}
\hline & OR (95\% Cl) & p Value \\
\hline sAA & $1.010(1.001$ to 1.018$)$ & 0.031 \\
Age & $1.105(1.012$ to 1.207$)$ & 0.027 \\
\hline sAA, salivary $\alpha$-amylase.
\end{tabular}

$95 \%$ CI 1.000 to 1.009 ). The ROC curve analysis for sAA discriminating between those with and without short-term adverse outcome showed area under the curve to be 0.715 (95\% CI 0.590 to 0.839 ) (figure 2).

\section{DISCUSSION}

This is the first report to indicate that sAA activity, a surrogate marker of SNS activity, is an independent predictor of malignant VA episodes in patients with STEMI. The onset and incidence of VA in the present study were similar to previous reports. ${ }^{134}$ The finding of lower serum potassium in the VA group was also consistent with the results of others. ${ }^{16}$ Although the pathways or mechanisms linking high initial sAA activity to arrhythmogenesis are not well known and should be explored in future research, it is hypothesised that some common pathophysiological processes operating in MI may lead to increased sAA activity and an increased hazard of VA. The present findings concur with previous studies implicating sympathetic overactivity in the mechanism of VA and SCD, especially in the setting of myocardial ischaemia. ${ }^{17} 18$

In relatively delayed VA (ie, after $48 \mathrm{~h}$ ) after AMI, nonuniform myocardial denervation resulting in $\beta$-receptor upregulation and catecholamine hypersensitivity in the infarct zone has been implicated as an important mechanism. Indeed, the coexistence of denervated and hyperinnervated areas in the myocardium after AMI would result in increased electrophysiological heterogeneity during sympathetic activation, and thus lead to VA. ${ }^{19} 20$ Plasma epinephrine concentration, measured for 1 week after AMI, is closely related to triggered VA. ${ }^{21}$ In VA developing immediately after AMI, enhanced sympathetic outflow from the central nervous system is supported by animal

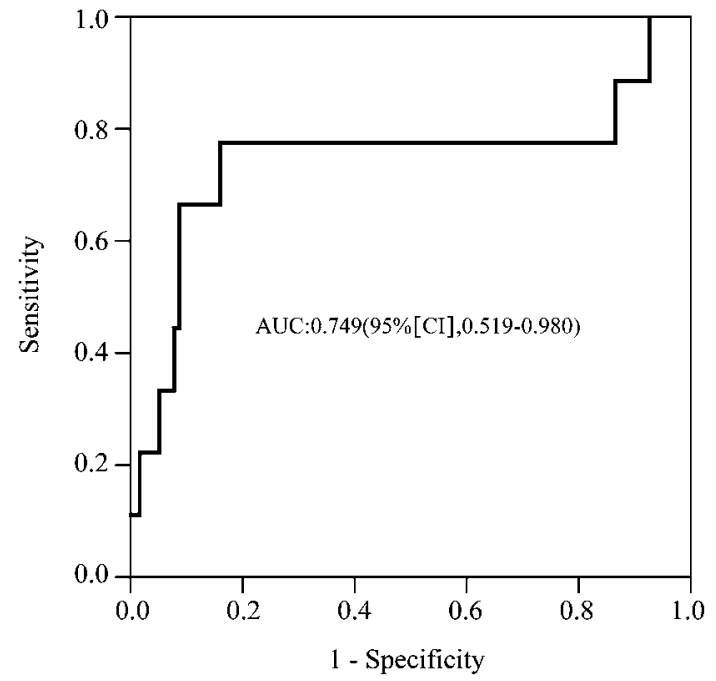

Figure 1 Receiver operating characteristic (ROC) curve of salivary $\alpha$ amylase (sAA) in predicting malignant ventricular arrhythmia in patients with ST-elevation myocardial infarction (STEMI). The area under the ROC curve (AUC) for sAA activity was 0.749 ( $95 \% \mathrm{Cl} 0.519$ to 0.980$)$.
Table 3 Demographic and clinical characteristics of the adverse outcome and no adverse outcome groups

\begin{tabular}{llll}
\hline & $\begin{array}{l}\text { Adverse } \\
\text { outcome } \\
\text { group (n=24) }\end{array}$ & $\begin{array}{l}\text { No adverse } \\
\text { outcome } \\
\text { group (n=67) }\end{array}$ & $\begin{array}{l}\text { p } \\
\text { value }\end{array}$ \\
\hline Age (years) & $63 \pm 11.2$ & $64 \pm 11.6$ & 0.804 \\
Gender, male/female, n (\%) & $18 / 6$ & $53 / 14$ & 0.775 \\
Smoker & $16(66.7)$ & $34(50.7)$ & 0.134 \\
Diabetes mellitus & $4(16.7)$ & $9(13.4)$ & 0.738 \\
Initial heart rate (bpm) & $84 \pm 18$ & $78 \pm 18$ & 0.309 \\
Laboratory data & & & 0.928 \\
$\quad$ WBC (per cm ${ }^{3}$ ) & $10.5 \pm 3.30$ & $10.6 \pm 3.06$ & 0.167 \\
$\quad$ Glucose (mg/dl) & $157 \pm 52.4$ & $152 \pm 73.0$ & 0.968 \\
Serum potassium (mEq/l) & $3.8 \pm 0.35$ & $3.8 \pm 0.36$ & $0.002^{*}$ \\
$\quad$ sAA activity (U/ml) & $334 \pm 81.0$ & $282 \pm 110.1$ & 0.627 \\
\hline Anterolateral infarction, $\mathrm{n}(\%)$ & $16(66.7)$ & $39(58.2)$ & \\
\hline
\end{tabular}

Continuous variables are presented as mean \pm SD.

Percent values are in parenthesis.

${ }^{*} p<0.05$ between two groups.

sAA, salivary $\alpha$-amylase; WBC, white cell.

studies, as well as clinical evidence, as a possible mechanism causing increased susceptibility to VA. ${ }^{5}$ For example, stress and exercise in animal models; the epidemiology of sudden death and its relationship to stress; the beneficial effect of $\beta$ blocker therapy in reducing the incidence of SCD after MI; and the similar circadian pattern of plasma catecholamine level, MI and VA all suggest a close association between early VA and increased sympathetic activity in patients with MI. ${ }^{17}{ }^{18}$ In patients with coronary artery disease and an implantable cardioverter defibrillator, sympathetic activation, as reflected by gradual increased heart rate, is the predominant change before a VA and may occur as early as an hour and a half before the arrhythmia. ${ }^{22}$ Sympathetic denervation of the ischaemic area may begin within minutes of the onset of ischaemia, creating electrophysiological heterogeneity between ischaemic and normal myocardium. ${ }^{5}$ Elevated epinephrine levels may enhance automaticity and triggered automaticity, as well as facilitate reentry to generate rhythm abnormalities. Additionally, the SNS can provoke the development of hypokalaemia and ischaemia

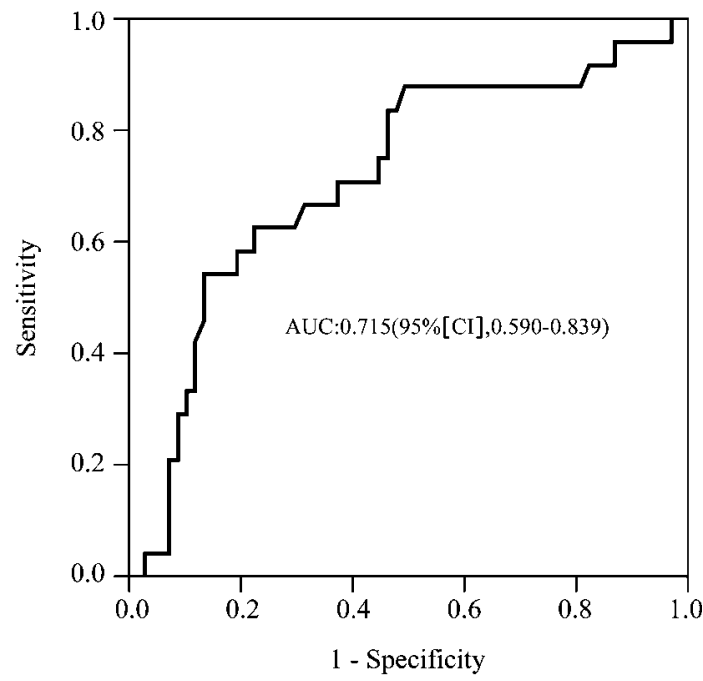

Figure 2 Receiver operating characteristic (ROC) curve of salivary $\alpha$ amylase (sAA) in predicting short-term prognosis in patients with STelevation myocardial infarction (STEMI). The area under the ROC curve (AUC) for sAA activity was 0.715 (95\% Cl 0.590 to 0.839$)$. 
(which can independently lead to the occurrence of rhythm disturbances), and catecholamines may negate the beneficial electrophysiological actions of antiarrhythmic drugs.

Sympathoadrenergic control of sAA secretion is well established. ${ }^{23}$ An association between sAA activity and autonomic cardiovascular parameters has been reported in healthy subjects in response to physical as well as psychological stressors. ${ }^{13}$ Furthermore, exercise, ${ }^{24}$ and smoking, ${ }^{25}$ as well as psychological factors known to stimulate sAA activity such as anger, ${ }^{26}$ anxiety, phobic anxiety ${ }^{27}$ and maladaptive behaviour in stressful encounters, ${ }^{28}$ have been shown to be associated with the risk of VA or SCD, providing a theoretical basis for the use of the sAA assay.

Different methodologies have been used to stratify the risk of VA after AMI. For example, biomarkers (brain natriuretic peptide, high sensitivity $C$ reactive protein) ${ }^{29}$ extent of coronary artery disease on coronary angiography; ${ }^{30}$ demographic factors and clinical features (eg, blood pressure, ${ }^{31}$ serum glucose level and white cell counts ${ }^{16} 32{ }^{33}$ ) as well as non-invasive electrocardiology parameters (late potentials, heart rate variability, baroreflex sensitivity, T wave alternance) and invasive electrophysiological methods (inducibility of ventricular tachycardia) have been used. These parameters all have limitations. The role of assessing SAA activity as a stand-alone risk stratification tool in the ED setting remains to be conclusively determined. However, sAA activity assay could potentially be incorporated with other assays into a model for assessing the risk of VA after MI.

It is also clear from the present study that high sAA activity in patients with STEMI is predictive of a poor short-term prognosis. The clinical presentation of AMI varies widely. Some patients have only a mild constitutional upset, whereas others suffer intense vasoconstriction, arrhythmias, heart failure or shock. The mechanisms for adverse outcome of AMI may involve the effects of stress hormones or the extent of the myocardial infarct itself. ${ }^{34} 35$ Plasma catecholamines have been reported to be associated with a poor cardiac outcome after AMI. ${ }^{34}$ Although stimulation of neuroendocrine systems may be an appropriate response to acute myocardial injury, neurotransmitters that promote vasoconstriction or tachycardia may also be harmful. High concentrations of catecholamines are frequently associated with the presence of heart failure, shock or $\mathrm{VA}$, and the potential for reducing infarct size and arrhythmias by interference with the catecholamine response by $\beta$ blockers has attracted much interest. High sAA activity levels probably reflect a high catecholamine surge, and thus the dire consequences associated with such change.

Simple logistic regression analysis showed that sAA is an independent predictor for short-term prognosis, suggesting that we could potentially characterise a group of patients who are at low risk for an adverse event during 1-month of follow-up. This novel finding is of significant clinical importance. Repeated risk assessment on regular basis that includes a sAA activity is of interest and should be studied.

Previous studies have demonstrated lower or similar incidences of VA in STEMI patients, with more than $90 \%$ of the events being recorded within the first $48 \mathrm{~h}$ after symptom onset. ${ }^{36-38}$ The present study further indicates that the majority of VA episodes occur while STEMI patients are still in the ED. As several multivariable models have failed to discriminate between patients with early malignant VA and those without, ${ }^{33}$ the clinical utility of the present findings lies in helping to identify STEMI patients in the ED who are high risk for VA and will benefit from an implantable cardioverter defibrillator or temporary pacing device. Especially for patients without previous experience of arrhythmic collapse, the survival benefit associated with such is substantial and the indication is costeffective. In the present study, sAA activity analysis was performed with frozen samples in batches using a commercially available kit in the laboratory. The recent invention of a portable automated sAA activity analyser may allow early stratification of high-risk patients in the ED possible. The device uses a disposable test-strip and measures the sAA activity with high accuracy with only $30 \mu \mathrm{l}$ sample of saliva within a minute from collection to completion of the measurement. ${ }^{39}$ Therefore, it will be possible to alert ED physicians of AMI patients with high risk of VA and to adopt preventive measures such as having defibrillator stand-by or start antiarrhythmic medications.

There were some notable limitations in the present study. There were potential confounding factors in measurement of sAA activity levels: variable baseline stress depending on personal habits, medications, lifestyle, personal fitness; psychological factors and underlying diseases. Residual confounding by these factors could account for at least part of the association between VA and sAA activity. However, as patients were not selected against these factors, it is hoped to balance the confounding effects in the non-VA group of patients. Future study with larger sample sizes could determine the effects of those confounders. Second, this analysis represents a singlecentre study with a relatively small number of patients and only focuses on the STEMI-type MI. It has been shown that the incidence of VA is relatively low in NSTEMI patients, about onequarter to one-third of the risk in patients with STEMI. ${ }^{33} 38$ However, patients with STEMI and NSTEMI have similar prognoses and independent correlates of outcome. ${ }^{40}$ For future study, comparison of sAA activity with long-term prognosis and VA occurrence including NSTEMI patients may provide more information and statistical power for clinical use. Third, the present analysis was based on a single determination of sAA activity, which does not allow assessment of potential changes of this SNS marker over time. Thus, this study would tend to obscure associations if the effect is of short duration. Lastly, although sAA activity is a non-invasive variable that the clinician can easily obtain for arrhythmia risk stratification in AMI patients, further prospective studies are needed to compare its predictive ability with that of previously defined non-invasive electrophysiological tests. Supplementary measurements of humoral status such as serum catecholamine levels would also add value in relating sympathetic activation and ventricular dysrhythmia.

\section{CONCLUSION}

Although prospective study with a large cohort is required, the present results suggest that high initial sAA activity is associated with increased risk of malignant VA and predicts short-term prognosis in patients with STEMI.

Acknowledgements We thank Drs Min-Hui Li and Cheng-Wen Ho for their helpful comments on this manuscript and statistician Yue-Cune Chang, PhD, for his professional assistance to the statistical analysis of this study.

Funding This study was supported, in part, by a grant from National Defense Medica Center D0D98-01-03, No. 161, Section 6, Min-Chuan East Road, Taipei, 114 Taiwan, R.O.C.

\section{Competing interests None.}

Ethics approval This study was conducted with the approval of the Cathay General Hospital, Taipei.

Contributors $\mathrm{HC}$ initiated and coordinated the formulation of the primary study hypothesis, discussed core ideas, designed the protocol and participated in data 
analysis and interpretation and writing of the paper. Y-SS, the first author of the manuscript, discussed core ideas, and participated in the protocol design, data collection, analysis, documentation and interpretation, and the writing of the paper. C-MC participated in the data documentation and interpretation, and contributed to the writing of the paper. W-LC participated in the data collection, documentation, analysis and interpretation, and critical review of the manuscript. J-HC and $\mathrm{H}-\mathrm{YC}$ participated in the design and execution of the study, particularly data collection and quality control.

Provenance and peer review Not commissioned; externally peer reviewed.

\section{REFERENCES}

1. Henkel DM, Witt BJ, Gersh BJ, et al. Ventricular arrhythmias after acute myocardial infarction: a 20-year community study. Am Heart $J$ 2006;151:806-12.

2. Campbell RW, Murray A, Julian DG. Ventricular arrhythmias in first 12 hours of acute myocardial infarction. Natural history study. Br Heart J 1981;46:351-7.

3. Thompson CA, Yarzebski J, Goldberg RJ, et al. Changes over time in the incidence and case-fatality rates of primary ventricular fibrillation complicating acute myocardial infarction: perspectives from the Worcester Heart Attack Study. Am Heart J 2000;139:1014-21.

4. Behar S, Reicher-Reiss H, Schechter M, et al. Frequency and prognostic significance of secondary ventricular fibrillation complicating acute myocardial infarction. SPRINT Study Group. Am J Cardiol 1993;71:152-6.

5. Podrid PJ, Fuchs T, Candinas R. Role of the sympathetic nervous system in the genesis of ventricular arrhythmia. Circulation 1990;82(2 Suppl):1103-13.

6. Sharma AD, Corr PB. Adrenergic factors in arrhythmogenesis in the ischemic and reperfused myocardium. Eur Heart $J$ 1983;4:12

7. Du XJ, Cox HS, Dart AM, et al. Sympathetic activation triggers ventricular arrhythmias in rat heart with chronic infarction and failure. Cardiovasc Res 1999:43:919-29.

8. Vanoli E, De Ferrari GM, Stramba-Badiale M, et al. Vagal stimulation and prevention of sudden death in conscious dogs with a healed myocardial infarction. Circ Res 1991;68:1471-81.

9. Kawada T, Yamazaki T, Akiyama T, et al. Vagal stimulation suppresses ischemiainduced myocardial interstitial norepinephrine release. Life Sci 2006;78:882-7.

10. Rissanen V, Romo M, Siltanen P. Premonitory symptoms and stress factors preceding sudden death from ischaemic heart disease. Acta Med Scand 1978;204:389-96

11. Reich P, Desilva RA, Lown B, et al. Acute psychological disturbances preceding lifethreatening ventricular arrhythmias. JAMA 1981:246:233-5.

12. Granger DA, Kivlighan $\mathrm{KT}$, Fortunato $\mathrm{C}$, et al. Integration of salivary biomarkers into developmental and behaviorally-oriented research: problems and solutions for collecting specimens. Physiol Behav 2007:92:583-90.

13. Nater UM, La Marca R, Florin L, et al. Stress-induced changes in human salivary alpha-amylase activity-associations with adrenergic activity.

Psychoneuroendocrinology 2006;31:49-58.

14. Thygesen K, Alpert JS, White HD. Joint ESC/ACCF/AHA/WHF Task Force for the Redefinition of Myocardial Infarction. Universal definition of myocardial infarction. Eur Heart J 2007;28:2525-38.

15. Hollander JE, Blomkalns AL, Brogan GX, et al. Standardized reporting guidelines for studies evaluating risk stratification of ED patients with potential acute coronary syndromes. Acad Emerg Med 2004;11:1331-40.

16. Gheeraert PJ, De Buyzere ML, Taeymans YM, et al. Risk factors for primary ventricular fibrillation during acute myocardial infarction: a systematic review and meta-analysis. Eur Heart J 2006;27:2499-510.

17. Zipes DP, Wellens HJ. Sudden cardiac death. Circulation 1998;98:2334-51.

18. Cornélissen G, Tamura $K$, Tarquini $B$, et al. Differences in some circadian patterns of cardiac arrhythmia, myocardial infarctions and other adverse vascular events. Chronobiologia 1994:21:79-88.
19. Chen LS, Zhou S, Fishbein MC, et al. New perspectives on the role of autonomic nervous system in the genesis of arrhythmias. J Cardiovasc Electrophysiol 2007:18:123-7.

20. Simóes MV, Barthel P, Matsunari I, et al. Presence of sympathetically denervated but viable myocardium and its electrophysiologic correlates after early revascularised acute myocardial infarction. Eur Heart J 2004;25:551-7.

21. Ejima J, Kaneko T, Maruyama T, et al. Increased plasma epinephrine but not reduced heart rate variability leads to ventricular arrhythmias in patients with acute myocardial infarction. J Cardiol 1998:32:69-75.

22. Copie $\mathbf{X}$, Lamaison D, Salvador $\mathbf{M}$, et al. Heart rate variability before ventricular arrhythmias in patients with coronary artery disease and an implantable cardioverter defibrillator. Ann Noninvasive Electrocardiol 2003:8:179-84.

23. Nater UM, Rohleder N. Salivary alpha-amylase as a non-invasive biomarker for the sympathetic nervous system: current state of research. Psychoneuroendocrinology 2009;34:486-96.

24. Elhendy A, Chandrasekaran K, Gersh BJ, et al. Functional and prognostic significance of exercise-induced ventricular arrhythmias in patients with suspected coronary artery disease. Am J Cardiol 2002;90:95-100

25. Gabbay FH, Krantz DS, Kop WJ, et al. Triggers of myocardial ischemia during daily life in patients with coronary artery disease: Physical and mental activities, anger and smoking. J Am Coll Cardiol 1996;27:585-92.

26. Lampert R, Joska T, Burg MM, et al. Emotional and physical precipitants of ventricular arrhythmia. Circulation 2002;106:1800-5.

27. Albert CM, Chae CU, Rexrode KM, et al. Phobic anxiety and risk of coronary heart disease and sudden cardiac death among women. Circulation 2005;111:480-7.

28. André-Petersson L, Engström G, Hedblad B, et al. Prognostic significance of ventricular arrhythmia modified by ability to adapt to stressful situations. Eur $J$ Cardiovasc Prev Rehabil 2004:11:25-32.

29. Blangy H, Sadoul N, Dousset B, et al. Serum BNP, hs-C-reactive protein, procollagen to assess the risk of ventricular tachycardia in ICD recipients after myocardial infarction. Europace 2007:9:724-9.

30. Hreybe $\mathbf{H}$, Singla I, Razak E, et al. Predictors of cardiac arrest occurring in the context of acute myocardial infarction. Pacing Clin Electrophysiol 2007;30:1262-6.

31. Yap YG, Duong T, Bland JM, et al. Prognostic value of blood pressure measured during hospitalization after acute myocardial infarction: an insight from survival trials. $J$ Hypertens 2007; 25:307-13.

32. Chen $\mathbf{J H}$, Tseng CL, Tsai SH, et al. Initial serum glucose level and white blood cell predict ventricular arrhythmia after first acute myocardial infarction. Am J Emerg Med 2010:28:418-23.

33. Rahimi K, Watzlawek S, Thiele $\mathrm{H}$, et al. Incidence, time course, and predictors of early malignant ventricular arrhythmias after non-ST-segment elevation myocardial infarction in patients with early invasive treatment. Eur Heart J 2006;27:1706-11.

34. Bain RJ, Fox JP, Jagger J, et al. Serum cortisol levels predict infarct size and patient mortality. Int J Cardiol 1992;37:145-50.

35. Vetter NJ, Strange RC, Adams W, et al. Initial metabolic and hormonal response to acute myocardial infarction. Lancet 1974;1:284-8.

36. Mehta RH, Starr AZ, Lopes RD, et al. Incidence of and outcomes associated with ventricular tachycardia or fibrillation in patients undergoing primary percutaneous coronary intervention. JAMA 2009;301:1779-89.

37. Newby KH, Thompson T, Stebbins A, et al. Sustained ventricular arrhythmias in patients receiving thrombolytic therapy: incidence and outcomes. The GUSTO Investigators. Circulation 1998;98:2567-73.

38. Newby LK, Hasselblad V, Armstrong PW, et al. Time-based risk assessment after myocardial infarction. Implications for timing of discharge and applications to medical decision-making. Eur Heart J 2003;24:182-9.

39. Yamaguchi M, Deguchi M, Wakasugi J, et al. Hand-held monitor of sympathetic nervous system using salivary amylase activity and its validation by driver fatigue assessment. Biosens Bioelectron 2006;21:1007-14.

40. Montalescot G, Dallongeville J, Van Belle E, et al. STEMI and NSTEMI: are they so different? 1 year outcomes in acute myocardial infarction as defined by the ESC/ACC definition (the OPERA registry). Eur Heart $J$ 2007:28:1409-17. 